\section{RECEIVED}

Alli 12 1997

OSTI
ANL-HEP-PR-94-77

December 2, 1994

\title{
Using Spin to Probe Hadronic Structure*
}

\author{
Gordon P. Ramsey \\ Physics Department, Loyola University Chicago, Chicago, IL 60626 \\ and \\ High Energy Physics Division, Argonne National Lab, Argonne, IL 60439
}

\begin{abstract}
The theoretical and experimental status of high energy spin phenomena is summarized, with emphasis on the spin properties of nucleons. It is stressed that crucial tests of the Standard Model can be made with polarization experiments. By performing the experiments discussed here, we will reveal important constituent and composite properties of protons and neutrons. The future prospects for planned polarization experiments are discussed.
\end{abstract}

\section{MASTER}

\section{DISCLAIMER}

This report was prepared as an account of work sponsored by an agency of the United States Government. Neither the United States Government nor any agency thereof, nor any of their employees, makes any warranty, express or implied, or assumes any legal liability or responsibility for the accuracy, completeness, or usefulness of any information, apparatus, product, or process disclosed, or represents that its use would not infringe privately owned rights. Reference herein to any specific commercial product, process, or service by trade name, trademark, manufacturer, or otherwise does not necessarily constitute or imply its endorsement, recommendation, or favoring by the United States Government or any agency thereof. The views and opinions of authors expressed herein do not necessarily state or reflect those of the United States Government or any agency thereof.

"Work supported by the U.S. Department of Energy, Division of High Energy Physics, Contract W-31109-ENG-38. Submitted to Particle World, c Gordon and Breach. 


\section{DISCLAIMER}

Portions of this document may be illegible in electronic image products. Images are produced from the best available original document. 


\section{Introduction}

Since the Stern-Gerlach experiment, it has been known that spin is one of the most fundamental properties of elementary particles. The property of spin allows selection of a "preferred" direction, which enables us to investigate the behavior of particles under spacetime transformations. By polarizing an accelerating beam of a fundamental particles, and colliding it with a target, we are able to investigate the "spin" properties of the fundamental building blocks of matter. Generally, there are two levels at which we probe hadronic matter: (i) at the constituent level and (ii) at the hadronic level. Different physics can be learned at each of these levels.

Theoretically, the constituent level effects are characterized by the spin dependent structure functions, which are a measure of the quark helicity current distributions with respect to light-cone momentum fraction, $x$. Naturally, the sum of the spins due to each of the constituents, added to the angular momentum of their aggregate should equal the total spin of the hadron, namely $\frac{1}{2}$. The magnetic moment of the hadron is a measure of the global spin properties of the constituents. In addition, total cross sections of polarized beams impinging on polarized or unpolarized targets can reveal properties of the whole hadron, especially in elastic scattering where the hadron retains its identity after the interaction.

Since cross sections are the most fundamental measured property of elementary particles, all of the theoretical quantities characterizing spin must be extracted from some combination of the polarization cross sections. In nature, asymmetric behavior often occurs between the interactions of particles whose spins are aligned and interactions of particles whose spins are anti-aligned. Thus, it is convenient to define an asymmetry as:

$$
A=\left[\frac{\sigma(++)-\sigma(+-)}{\sigma(++)+\sigma(t-)}\right]
$$

where the ++ refers to the aligned spins and +- to the anti-aligned spins, without regard to the direction of polarization relative to the momentum of the interaction. There are distinct advantages to defining the asymmetries in this way. Theoretically, $A$ is a ratio of cross sections, so all of the normalizations used to calculate cross sections cancel, leaving only the key parameters of the interaction. Experimentally, the difference in the numerator tends to cancel some of the systematic errors, making $A$ a fundamentally more sensitive measure of the physical parameters.

Significant interest in high energy spin physics was piqued a few years ago when the European Muon Collaboration (EMC) ${ }^{1}$ published polarized deep-inelastic scattering data which appeared to contradict theoretical predictions, creating the "spin crisis". Since then, a flurry of theoretical and experimental work has been performed to clarify this "crisis" and 
further investigate the spin properties of the lighter hadrons. In section 2, I give an overview of the present experimental status of high energy polarization physics. Section 3 will include explanations of the basic constituent spin structure functions and what is presently known about them. In section 4, the theoretical aspects of the hadronic level spin properties will be discussed. In section 5, I will give an overview of some proposed polarization experiments at different accelerators, with an emphasis on the physics that can be extracted from them.

\section{Present Experimental Status}

\subsection{Introduction}

High energy polarization experiments were essentially begun in the 1970's at the Argonne ZGS with the measurement of the polarization asymmetry, $A_{N} .{ }^{2}$ This represented one of the first times that a polarized target was used in a high energy ( $\gg 1 \mathrm{GeV}$ ) experiment. Shortly thereafter, other polarized beam and target experiments were performed at various accelerators, covering a wide range of available energies and momenta. ${ }^{3,4}$ Most of the early experiments yielded results which were unexpected, thus giving an impetus to further study of polarization phenomena. ${ }^{5}$

At this writing, there is a reasonable selection of polarization data at high energies, which has helped theorists define the questions to be answered regarding the spin content of nucleons. We have simultaneously discovered that many more experiments must be performed to answer these questions. In the following sections, I will outline some of the key questions that have arisen regarding nucleon spin and give a perspective on how the present data and how future polarized beam experiments play a role in answering these questions.

The easiest way to categorize the existing polarization data is via the categories of: (i) inclusive scattering and (ii) exclusive $p p$ (elastic) scattering. Generally, the polarized inclusive scattering experiments probe the inner structure of nucleons, giving spin information about their constituents. Meanwhile, the elastic experiments reveal the composite spin properties of nucleons as a group of valence quarks with spin quantum numbers, confined by an unknown mechanism. Polarization experiments are designed to help answer both the constituent and composite questions about nucleon structure. Experiments which are in the inclusive category include: (i) deep-inelastic scattering of polarized leptons $(e, \mu)$ on polarized nucleon targets $(p, n, d)$; (ii) production of jets in high energy polarized $p p$ and $p \bar{p}$ scattering; (iii) production of pions and direct photons in high energy polarized $p p$ and $p \bar{p}$ scattering; and (iv) lepton pair production (Drell-Yan) in polarized processes. Elastic processes consist primarily of polarized $p p$ scattering, carried out at a wide range of energies and beam momenta, and detected at a large range of angles. 


\subsection{Existing data}

One of the first polarized inclusive measurements made was by the SLAC/Yale group, ${ }^{3}$ which measured the spin dependent asymmetry in the deep-inelastic scattering of longitudinally polarized leptons on longitudinally polarized protons. This asymmetry is given by

$$
\begin{aligned}
A & =\left[\frac{\sigma(\hookleftarrow)-\sigma(\leftarrow)}{\sigma(\hookleftarrow)+\sigma(\leftarrow)}\right] \\
& =D\left(A_{1}+\eta A_{2}\right),
\end{aligned}
$$

where the arrows refer to the relative longitudinal spin directions of the beam and target, respectively. The terms $D$ and $\eta$ are known kinematic factors. Information about the polarized quark distributions can be extracted from this asymmetry by

$$
A_{1}=\frac{\sum_{i} e_{i}^{2} \Delta q_{i}(x)}{\sum_{i} e_{i}^{2} q_{i}(x)}
$$

where, for each quark flavor $i$ :

$$
\Delta q_{i}\left(x, Q^{2}\right) \equiv q_{i}^{+}\left(x, Q^{2}\right)-q_{i}^{-}\left(x, Q^{2}\right) .
$$

Here, the $+(-)$ indicates the quark spin aligned (anti-aligned) with the nucleon spin. The assumption is made that $A_{2}$ is small, since it is bounded by $R=\sqrt{ } \frac{\sigma_{L}}{\sigma_{T}}$, the ratio of cross sections for absorption of longitudinal and transverse virtual photons. This is an accurate assumption in the kinematic range of this data. The proton structure function $g_{1}^{p}$ can be extracted from the asymmetry $A_{1}$ by using

$$
g_{1}^{p}\left(x, Q^{2}\right) \approx \frac{A_{1}(x) F_{2}\left(x, Q^{2}\right)}{2 x(1+R)} .
$$

Here it is assumed that the transverse structure function $g_{2}^{p}$ is small and that $A_{1}$ is relatively independent of $Q^{2}$, an assumption which has been verified by experimental measurements. These measurements were done to extract information about the quark model picture of proton spin and to investigate a limit on parity non-conservation in polarized deep-inelastic scattering (DIS). Later, the European Muon Collaboration (EMC) at CERN extended the kinematic range of the DIS measurements to smaller Bjorken $x$ using a polarized muon beam. ${ }^{1}$ The extrapolation of the structure function $g_{1}^{p}$ to lower $x$ led to implications that, although the Bjorken sum rule of $\mathrm{QCD}^{6}$ was satisfied, the Ellis-Jaffe sum rule ${ }^{7}$ based on a simple quark model was violated. This created a controversy which heightened the interest in spin phenomena. This will be discussed in section 3. In the last couple of years, the Spin Muon Collaboration (SMC) group from CERN ${ }^{8}$ and the E142/E143 experimental 
groups from SLAC ${ }^{9}$ have measured $A_{1}^{p}$ and $g_{1}^{p}$ to even lower $x$ values and have added the corresponding neutron and deuteron structure functions $A_{1}^{n}, g_{1}^{n}, A_{1}^{d}$, and $g_{1}^{d}$. These groups have also improved statistics and lowered the systematic errors from the original data.

Recently, the E704 group at Fermilab has analyzed the data from their $p p$ and $p \bar{p}$ experiments for jet production cross sections. ${ }^{10}$ The results have implied that the polarized gluon distribution is limited in size, but has not put a strict value on this limit. In all of the inclusive experiments done this far, we have gained some knowledge of the constituent spin content, but it is clear that much more work has to be done in future experiments to obtain a complete understanding of this important problem in physics.

Unpolarized elastic scattering experiments have been done for many years. In the mid 1960 's through the early 1980 's, differential cross sections, $\frac{d \sigma}{d t}$, for $p p$ elastic scattering were measured for a wide variety of energies and beam momenta at the Argonne ZGS, ${ }^{11}$ the Brookhaven AGS, ${ }^{12}$ and the CERN ISR. ${ }^{13}$ These experiments provided a basis for analyzing the composite nature of protons. Later, at the ZGS, polarized beam experiments were performed to measure spin asymmetries. ${ }^{4}$ In particular, the double spin (beam and target polarized ) asymmetry $A_{N N}$ was measured at $90^{\circ} \mathrm{c.m}$. and for various fixed $p_{\text {lab }}$ (beam momenta). The oscillations in both the differential cross sections and the asymmetries have led to considerable controversy regarding the applicability of perturbative QCD (PQCD) to exclusive processes. ${ }^{14}$ Measurement of the polarization $A_{N}$ at the $\mathrm{ZGS}^{2}$ yielded a non-zero value, which lead to the speculation that helicity (the component of spin in the direction of the proton momentum) conservation at the hadronic level is questionable. In short, existing polarization data has helped to define the appropriate questions to ask regarding the nature of nucleon spin. In the next two sections, I will discuss the theoretical aspects of this topic.

\section{Constituent Spin and Inclusive Reactions}

\subsection{Introduction}

One of the important questions in high energy spin physics is how the spin of hadrons, like nucleons, is related to the spins of their quark and gluon constituents. There have been many approaches to this problem. ${ }^{15}$ Fundamentally, we assume that the nucleons are comprised of valence quarks (uud for the proton and udd for the neutron), which carry most of the quantum numbers of the nucleon and whose polarized distribution and integrated polarized distribution are defined by:

$$
\begin{aligned}
\Delta q_{v}\left(x, Q^{2}\right) & \equiv q_{v}^{+}\left(x, Q^{2}\right)-q_{v}^{-}\left(x, Q^{2}\right) \\
\left\langle\Delta q_{v}\left(Q^{2}\right)\right\rangle & \equiv \int_{0}^{1} \Delta q_{v}\left(x, Q^{2}\right) d x
\end{aligned}
$$


where $+(-)$ indicates the quark spin aligned (anti-aligned) with the nucleon spin. In addition, the nucleon is filled with a quark sea, which is polarized by gluon Bremsstrahlung and by quark-antiquark pair creation. The corresponding integrated polarized sea distribution is defined as:

$$
\left\langle\Delta S\left(Q^{2}\right)\right\rangle \equiv\left\langle\left[\Delta u\left(Q^{2}\right)+\Delta \bar{u}\left(Q^{2}\right)+\Delta d\left(Q^{2}\right)+\Delta \bar{d}\left(Q^{2}\right)+\Delta s\left(Q^{2}\right)+\Delta \bar{s}\left(Q^{2}\right)\right]\right\rangle .
$$

Here it is assumed that the lightest flavors dominate the spin properties, as the much heavier quarks would be significantly harder to polarize. In terms of the proton wave function, we can write these integrated distributions as

$$
\left\langle\Delta q_{i} s^{\mu}\right\rangle=\left\langle p s\left|\bar{q} \gamma^{\mu} \gamma_{5} q_{i}\right| p s\right\rangle / 2 m,
$$

where $s^{\mu}(p)$ is the axial four-vector which characterizes a spin $\frac{1}{2}$ particle and $m$ is the mass of the particle.

The gluons are polarized through Bremsstrahlung from the quarks. The integrated polarized gluon distribution is written as

$$
\langle\Delta G\rangle=\int_{0}^{1} \Delta G\left(x, Q^{2}\right) d x=\int_{0}^{1}\left[G^{+}\left(x, Q^{2}\right)-G^{-}\left(x, Q^{2}\right)\right] d x,
$$

where the $+(-)$ indicates spin aligned (anti-aligned) with the nucleon, as in the quark distributions.

There are natural constraints to these polarized distributions. For example, due to $\mathrm{O}(2)$ invariance, a proton with momentum and spin in the z-direction will conserve $J_{z}$. This total spin sum rule can be written in terms of the polarized distributions as:

$$
J_{z}=\frac{1}{2}=\frac{1}{2}\left\langle\Delta q_{v}\left(x, Q^{2}\right)\right\rangle+\frac{1}{2}\left\langle\Delta S\left(x, Q^{2}\right)\right\rangle+\langle\Delta G\rangle+L_{z} .
$$

The right hand side represents the decomposition of the constituent spins along with their relative angular momentum, $L_{z}$. Other constraints are provided by the axial-vector current operators, $A_{\mu}^{k}$, whose matrix elements for the proton define coefficients, $a^{k}$, as ${ }^{16}$ :

$$
\left\langle p s\left|A_{\mu}^{k}\right| p s\right\rangle=s_{\mu} a^{k},
$$

where the $a^{k}$ are non-zero for $\mathrm{k}=0,3$ and 8 . These current operators are members of an $\mathrm{SU}(3)_{f}$ octet, whose non-zero elements give relations between the polarized distributions and the measurable coefficients $a^{k}$. The Bjorken sum rule $e^{6}$ relates the polarized structure function $g_{1}(x)$, measured in polarized deep-inelastic scattering, to the axial vector current 
$A_{\mu}^{3}$. The coefficient $a^{3}$ is measured in neutron beta decay and this sum rule is considered to be a fundamental test of QCD. The Bjorken sum rule is normally written as

$$
\int_{0}^{1}\left[g_{1}^{p}(x)-g_{1}^{n}(x)\right] d x=a^{3}\left(1-\frac{\alpha_{s}}{\pi}+\cdots\right) .
$$

In terms of the polarized distributions it can be reduced to the form:

$$
\int_{0}^{1}\left[\Delta u\left(x, Q^{2}\right)-\Delta d\left(x, Q^{2}\right)\right] d x=a^{3}\left(1-\frac{\alpha_{s}}{\pi}+\cdots\right) .
$$

The coefficient $a^{8}$ is determined by hyperon decay, reflecting the other baryon axial charges in the symmetry. In terms of the quark polarized distribution functions, this relation can be written as:

$$
\int_{0}^{1}\left[\Delta u\left(x, Q^{2}\right)+\Delta d\left(x, Q^{2}\right)-2 \Delta s\left(x, Q^{2}\right)\right] d x=a^{8} .
$$

There is no direct measurement from $\beta$ decay for $a^{0}$, but it is related to the total spin carried by the quarks in the proton. This will be discussed in more detail later.

Although the spin is the important parameter in this discussion, when analyzing experimental data for longitudinally polarized particles, helicity is the more appropriate property to consider. This is defined as the component of the constituent's spin in the direction of the proton's momentum. Most of the discussion in this section will concentrate on the properties of the constituents in terms of helicity.

\subsection{Valence Quark Distributions}

In order to construct the polarized quark distributions from the unpolarized ones, we can start with a modified 3-quark model based on an SU(6) wave function for the proton. This model is based on flavor symmetry of the $u$ - and d-sea and constructs the valence distributions to satisfy the Bjorken sum rule. Since $A_{1}$ and $g_{1}^{p}$ are related to the polarized quark distributions, we can extract information about the valence distributions from the data. In particular, as $x \rightarrow 1$, the sea distributions are negligible and the valence quark distributions must predict the asymptotic value of $A_{1}$ The value given by the SLAC/Yale data $^{3}$ indicated that the modified model for valence quarks was too small. Thus, models by Carlitz and Kaur ${ }^{17}$ and separately by Qiu, et. al., ${ }^{15}$ among others, ${ }^{18}$ made physical justification for the valence quarks carrying most of the proton helicity only at large $x$ values. The modified valence quark distributions have the form:

$$
\begin{aligned}
& \Delta u_{v}\left(x, Q^{2}\right)=\cos \theta_{D}\left[u_{v}\left(x, Q^{2}\right)-\frac{2}{3} d_{v}\left(x, Q^{2}\right)\right], \\
& \Delta d_{v}\left(x, Q^{2}\right)=-\frac{1}{3} \cos \theta_{D} d_{v}\left(x, Q^{2}\right),
\end{aligned}
$$


where $\cos \theta_{D}$ is a "spin dilution" factor which vanishes as $x \rightarrow 0$ and becomes unity as $x \rightarrow 1$, characterizing the valence quark helicity contribution to the proton. Normally, the spin dilution factor is adjusted to satisfy the Bjorken sum rule and to agree with the deepinelastic data at large $x$. Using equation (13) and assuming flavor symmetry of the sea, the total spin carried by valence quarks is found to be about $75 \%$. This is in agreement with other theoretical approaches to quark spin. ${ }^{19}$

\subsection{Sea Distributions}

The helicity of the sea distributions can be structured to a first approximation by considering that the sea quarks obtain their polarization by gluons emitted by valence quarks, which in turn create sea quark-antiquark pairs. In the approach of Close and Sivers, ${ }^{20}$ the behavior of the valence quarks follows the quark counting rules. ${ }^{21}$ Then, the behavior of the polarized sea as a function of $x$ follows from the QCD diagrams for quark-antiquark creation. This model must be refined in order to account for the EMC results, ${ }^{1}$ which imply that the sea is polarized opposite of that to the proton. To refine the original model, we can first break the SU(6) symmetry of the sea by assuming that the heavier strange sea quarks will be harder to polarize. Then, we assume that the sea distributions are related in the following manner: 15

$$
\begin{aligned}
\Delta \bar{u}\left(x, Q^{2}\right) & =c(x) \Delta u\left(x, Q^{2}\right)=\Delta \bar{d}\left(x, Q^{2}\right)=c(x) \Delta d\left(x, Q^{2}\right) \\
& =(1+\epsilon(x)) \Delta \bar{s}\left(x, Q^{2}\right)=c(x)(1+\epsilon(x)) \Delta s\left(x, Q^{2}\right) .
\end{aligned}
$$

The $\epsilon(x)$ factor is a measure of the increased difficulty in polarizing sea quarks. The $c(x)$ term represents the possible enhancement of polarized antiquarks in the sea due to the asymmetry of spin-spin forces in the $q q$ and $q \bar{q}$ channels. The assumptions of equation (16) are consistent with the unpolarized data of the $\mathrm{NMC}^{22}$ which measured an enhancement of $\bar{d}$ quarks over $\bar{u}$ quarks in the proton. One has the option to similarly modify the relation between polarized flavors and their unpolarized counterparts, in case that future polarized data indicate a similar result. Note that this form for the polarized sea is consistent with the form of the Bjorken sum rule written as in equation (13). In order to determine the variables $\epsilon(x)$ and $c(x)$ and the overall size of the polarized sea, the structure functions measured in deep-inelastic polarized scattering, $g_{1}^{p}$, can be used.

The integrated polarized structure function is related to the polarized quark distributions by

$$
\int_{0}^{1} g_{1}^{p}(x) d x=\frac{1}{2}\left(1-\frac{\alpha_{s}}{\pi}\right)\left\langle\left[\frac{4}{9} \Delta u_{v}+\frac{1}{9} \Delta d_{v}+\frac{4}{9}\left(\Delta u_{s}+\Delta \bar{u}\right)+\frac{4}{9}\left(\Delta d_{s}+\Delta \bar{d}\right)+\frac{1}{9}(\Delta s+\Delta \bar{s})\right]\right\rangle .
$$

Thus, the data on $g_{1}^{p}$ can be used to extract information about the sea quark distributions. This is not enough, however, to determine all of the sea parameters. Additional constraints 
are provided by a traditional analysis of hyperon decays, which determine two empirical constants: $D$ and $F .^{23}$ These are related to the polarized quark distributions by the axial current $a^{8}$ mentioned above:

$$
a^{8}=\left\langle\left[\Delta u_{v}+\Delta d_{v}+\Delta u_{s}+\Delta \bar{u}+\Delta d_{s}+\Delta \bar{d}-2 \Delta s-2 \Delta \bar{s}\right]\right\rangle=3 F-D \approx 0.58 \pm 0.02 .
$$

As Lipkin has pointed out, one must proceed with caution in using hyperon spin structures, however, without a suitable hyperon spin model. Since the weak decays measure flavorchanging transition matrix elements, which depend on both initial and final state wave functions, difficulties arise in trying to fit hyperon spin models with the observed magnetic moments and weak decays. ${ }^{24}$ Thus, although we can use the above equation for $a^{8}$ to extract information about the quark sea, there must be alternate consistency tests of these sea models in order to verify their validity.

As previously mentioned, the total spin carried by quarks is related to the axial current, $a^{0}$. In particular, we can relate the non-zero axial currents and the structure function $g_{1}^{p}$ in the following way:

$$
a^{0} \approx 9\left(1+\frac{\alpha_{s}}{\pi}\right) \int_{0}^{1} g_{1}^{p}(x) d x-\frac{1}{4} a^{8}-\frac{3}{4} a^{3} .
$$

Thus, the equations for the axial current coefficients $a^{i}$ give constraints to the polarized quark distributions, from which we can attempt to extract specific information about individual contributions to the overall proton spin. Shortly after the EMC experiment, there were a number of theoretical calculations which isolated the contributions of each of the flavors of the polarized sea to the proton's helicity. ${ }^{15,25}$ All of these came to the conclusion that the sea is negatively polarized. This is a reasonable conclusion when one takes into account the spin dependent forces which cause polarization of the sea from valence quarks and gluons. ${ }^{15}$ With the advent of the recent SMC and SLAC experiments, ${ }^{8-9}$ modified values for the flavors of polarized distributions have been given. ${ }^{23,26}$ Results for some of the polarized sea distributions, based on various models and data, are shown in Table 1. The models are keyed as follows: (i) QRRS- models by Qiu, et. al., ${ }^{15}$ based on the EMC data and on a valence dominated model (VDM) which limits the strange sea; (ii) BEK- the model by Brodsky, et. al., ${ }^{25}$ based on the EMC data; (iii) HJL- Lipkin's model III ${ }^{25}$ which sets the strange sea contribution to zero and bases the rest on the EMC data; (iv) models by Close and Roberts ${ }^{23}$ , based on SMC and SLAC data ${ }^{8-9}$ for the proton $\left(I_{p}\right)$, neutron $\left(I_{n}\right)$ and the deuteron $\left(I_{d}\right)$; (v) models by Goshtasbpour and Ramsey ${ }^{26}$, also based on SMC and SLAC data for the proton, neutron and the deuteron, with a VDM model as in reference 15. 


\section{Table 1}

$\begin{array}{ccc}\underline{\text { Model }} & \underline{\Delta u=\Delta d} & \underline{\Delta s} \\ Q R R S(E M C) & -0.24 & -0.15 \\ Q R R S(V D M) & -0.10 & -0.01 \\ B E K & -0.26 & -0.23 \\ H J L(I I I) & -0.27 & +0.00 \\ C R\left(I_{p}\right) & -0.21 & -0.12 \\ C R\left(I_{n}\right) & -0.12 & -0.03 \\ C R\left(I_{d}\right) & -0.20 & -0.11 \\ G R\left(I_{p}\right) & -0.19 & -0.09 \\ G R\left(I_{n}\right) & -0.10 & -0.01 \\ G R\left(I_{d}\right) & -0.25 & -0.15 \\ G R(V D M) & -0.10 & -0.01\end{array}$

It can be seen that there is some consistency between certain models, but that a wide variety of values for each flavor are possible, depending on which theoretical assumptions or experimental data are used to determine the values. All of thse are compatible with some of the data, but there are still inconsistencies with the various sets of data. Thus, it is clear that more tests are necessary. As pointed out by Qiu, et. al., ${ }^{15}$ the most direct experiment to determine the size of the polarized sea is lepton pair production (Drell-Yan) processes in polarized nucleon scattering experiments. Only then will there be enough information to tell which assumptions about the polarized sea are appropriate. ${ }^{27}$

\subsection{Gluon Distribution Models}

We cannot infer a priori the size of the polarized gluon distribution in a proton at a given $Q^{2}$ value. From the evolution equations for the polarized distributions, we do know that the polarized gluon distribution increases with $Q^{2}$ and that its evolution is directly related to the behavior of the orbital angular momentum, since the polarized quark distributions do not evolve in $Q^{2}$ in leading order. ${ }^{15}$ Thus, the normal approach is to assume a particular distribution for a given $Q^{2}$ and check the consistency of the assumptions with experimental data which is sensitive to $\Delta G\left(x, Q^{2}\right)$ at a particular $Q_{0}^{2}$. Initial analyses of the EMC data ${ }^{25}$ led to speculation that the integrated gluon distribution may be quite large, even at the relatively small value of $Q^{2}=10.7 \mathrm{GeV}^{2}$. In the models of Qiu, et. al., ${ }^{15}$ and Ramsey 
and Sivers ${ }^{28}$, three separate scenarios involving different sized polarized gluon distributions were investigated at the average $Q^{2}$ of the EMC data. The first assumes effectively no gluon polarization at $Q_{0}^{2}=10.7 \mathrm{GeV}^{2}$. The second model was motivated by the spin-weighted evolution equations, whose splitting functions contain an additional $x$ factor. The resulting Ansatz is:

$$
\Delta G\left(x, Q^{2}\right)=x G\left(x, Q^{2}\right),
$$

whose integrated distribution gives about 0.5 . The third model was created to extremize the integrated gluon distribution to the value of about 5.0, consistent with some of the early analyses of the EMC results. This model assumes that

$$
\begin{aligned}
& \Delta G\left(x, Q^{2}\right)=\left(\frac{x}{x_{0}}\right) G\left(x, Q^{2}\right), \quad x \leq x_{0}, \\
& \Delta G\left(x, Q^{2}\right)=G\left(x, Q^{2}\right), \quad x \geq x_{0}
\end{aligned}
$$

where $x_{0}$ is a variable cutoff used to determine the actual value of $\int_{0}^{1} \Delta G(x) d x$. Subsequent experiments and analyses have favored the models giving smaller integrated values for $\Delta G{ }^{10}$

Since the polarized gluon distribution does not explicitly appear in the axial currents mentioned above, separate experiments must be performed to indicate its value at various $Q^{2}$. There have been a number of papers which indicate some of the appropriate experiments that are sensitive to $\Delta G$. ${ }^{28-32}$ Among these include: (i) jet production, ${ }^{28-30}$ (ii) direct photon $(\gamma)$ production, ${ }^{28,31}$ and (iii) pion production ${ }^{28,32}$ in polarized $p p$ and $p \bar{p}$ collisions, where both the target and beam are polarized. All of these are extremely sensitive to the polarized gluon distribution, especially at high energies and small $x$, where the gluons are most prominent.

The model of $\Delta G$ which is finally determined by experiments has a direct effect on the actual value of the quark distributions through the gluon axial anomaly. ${ }^{33}$ In QCD, the U(1) axial current matrix element $A_{\mu}^{0}$ is not strictly conserved, even with massless quarks. Hence, at two loop order, the triangle diagram between two gluons generates a $Q^{2}$ dependent gluonic contribution to the measured polarized quark distributions. This term has the general form:

$$
\Gamma\left(Q^{2}\right)=\frac{N_{f} \alpha_{s}\left(Q^{2}\right)}{2 \pi} \int_{0}^{1} \Delta G\left(x, Q^{2}\right) d x
$$

where $N_{f}$ is the number of quark flavors. Thus, for each flavor of quark appearing in the distributions, the measured polarization distribution is modified by a factor: $\left\langle\Delta q_{i}\right\rangle-$ $\Gamma\left(Q^{2}\right)$. In order for us to determine the quark contributions to the spin of the nucleons, it is necessary for us to know the relative size of the polarized gluon distribution. Certainly, many more experiments of the types listed above must be performed in order to determine these important parameters. This will be discussed in more detail in part 5 . While constituent 
contributions to nucleon spin are important, it is also interesting to investigate the spin properties of nucleons as whole entities. Elastic scattering experiments play a vital role in this study.

\section{Hadronic Spin and Exclusive Reactions}

\subsection{Introduction}

One of the main goals of QCD is to understand the overall structure of hadrons and the nature of short interaction dynamics. Since hadrons remain intact in elastic scattering, exclusive reactions have been studied extensively to achieve these goals. For twenty years, polarized elastic scattering has provided a rich set of problems, some of which still remain a mystery. ${ }^{2,4,11}$ There are many fundamental physics questions which can be investigated by studying hadronic spin. In a recent article in this journal, 34 Troshin and Tyurin outlined some of these questions. I will review three of these in light of recent theoretical work done in this area: ${ }^{35}$ (i) normalization of the proton wave function, which is crucial in determining the predictive power of perturbative QCD (PQCD) in exclusive processes, (ii) helicity conservation on the hadronic level, which sheds light on chiral symmetry breaking and (iii) non-perturbative QCD (NPQCD) effects, which may be able to explain some of the mysteries which present data imply. All of these important questions are testable using polarized exclusive processes.

\subsection{Wave Function Normalization}

The hadronic wave function is normally decomposed into a sum over n-parton Fock states, whose overall normalization is not known. In normal elastic binary processes $(A+B \rightarrow$

$C+D)$, the valence quark wave functions determine the hadronic interaction process. ${ }^{36}$ For $p p \rightarrow p p$ hard scattering processes, there are many difficulties in performing a "first principles" calculation of the observable quantities. First, there are about 300,000 distinguishable Feynman diagrams, so that considerable computing power is required to even begin to categorize and evaluate them. Then there are the PQCD questions of renormalization group dependence and factorization prescription of the perturbative terms in the calculation. Finally, the definition of the wave function affects normalizations of endpoint and pinch singularities, which can result in uncertain normalization in individual scattering amplitudes which contribute to these processes. Thus, the most practical approach to this problem is from a phenomenological point of view. ${ }^{35}$

There are generally two types of diagrams which contribute to hard scattering $p p$ processes. The first are those diagrams in which the constituent scatterings occur in a pointlike 
region and therefore satisfy the dimensional counting rules for the differential cross section ${ }^{21}$

$$
\frac{d \sigma}{d t}(A+B \rightarrow C+D) \approx F(\theta) s^{2-n_{A}-n_{B}-n_{C}-n_{D}},
$$

where $F(\theta)$ is a kinematic factor and the $n_{i}$ are the number of constituent participants in the " $i$ " hadron. The other type of diagrams, introduced by Landshoff,, $37-38$ are characterized by multiple scatterings by the constituents over a non-pointlike region. This behavior modifies the exponent in the counting rule behavior for $p p$ elastic scattering to 9.59 , instead of 10 , as in the pointlike scattering. ${ }^{39}$

In order to calculate the spin observables for elastic scattering, we write them in terms of the Jacob-Wick helicity amplitudes ${ }^{40}$

$$
\begin{aligned}
& \Phi_{1}(s, t)=\langle++|M|++\rangle, \\
& \Phi_{2}(s, t)=\langle++|M|--\rangle, \\
& \Phi_{3}(s, t)=\langle+-|M|+-\rangle, \\
& \Phi_{4}(s, t)=\langle+-|M|-+\rangle, \\
& \Phi_{5}(s, t)=\langle++|M|--\rangle .
\end{aligned}
$$

Other helicity amplitudes are related to these using parity conservation, time reversal invariance and identical particle symmetry. The differential cross section is given in terms of these amplitudes by:

$$
\frac{d \sigma}{d t}=\frac{\pi}{2 s\left(s-4 m^{2}\right)}\left[\left|\Phi_{1}\right|^{2}+\left|\Phi_{2}\right|^{2}+\left|\Phi_{3}\right|^{2}+\left|\Phi_{4}\right|^{2}+4\left|\Phi_{5}\right|^{2}\right]
$$

while the other spin asymmetries are written as:

$$
\begin{aligned}
\sigma & =s\left(s-4 m^{2}\right) \frac{d \sigma}{d t} \\
A_{N} \sigma & =-\Im\left[\Phi_{5}^{*}\left(\Phi_{1}+\Phi_{2}+\Phi_{3}-\Phi_{4}\right)\right] \\
A_{S L} \sigma & =\Re\left[\Phi_{5}^{*}\left(\Phi_{1}+\Phi_{2}-\Phi_{3}+\Phi_{4}\right)\right] \\
A_{N N} \sigma & =\Re\left[\Phi_{1} \Phi_{2}^{*}-\Phi_{3} \Phi_{4}^{*}+2\left|\Phi_{5}\right|^{2}\right] \\
A_{S S} \sigma & =\Re\left[\Phi_{1} \Phi_{2}^{*}+\Phi_{3} \Phi_{4}^{*}\right] \\
A_{L L} \sigma & =\frac{1}{2}\left[-\left|\Phi_{1}\right|^{2}-\left|\Phi_{2}\right|^{2}+\left|\Phi_{3}\right|^{2}+\left|\Phi_{4}\right|^{2}\right] .
\end{aligned}
$$

Here the asymmetries are defined as in equation (1), with the directions of spin given as: $L$ for the longitudinal or "helicity" direction, and $N$ and $S$ for the normal and sideways 
(transverse components) spins respectively. In the hard scattering region, it is assumed that we can separate out the soft "Regge" contributions from those of the Landshoff diagrams and those diagrams satisfying the counting rules. Farrar, et.al. and Brodsky, et.al. ${ }^{41}$ have calculated the cross section angular factors for the counting rule diagrams, hereafter refered to as Quark Interchange Mechanism (QIM) contributions. We can therefore write the amplitudes as a sum of QIM and Landshoff contributions, each with their own characteristic normalizations. Since the asymmetries are ratios of these amplitudes, we can factor out an overall normalization constant, which cancels in the ratio, and write the observables in terms of a relative normalization between the QIM and Landshoff diagrams. This relative normalization is sensitive to the kinematics (in particular, the Mandelstam variables $s$ and $t$ ) and is a measure of the relative importance of the two contributions.

In order to extract a phenomenological expression for the overall wave function normalization, we can combine knowledge gained from the asymmetries and the cross sections, which depend on the overall normalization. Our most recent analysis ${ }^{35}$ of the $90^{\circ} \mathrm{c.m}$. data at the ANL ZGS,11 indicate that non-perturbative effects likely contribute to the wave function, even at large values of the momentum transfer, $-t$. Clearly, more data are needed at larger $\sqrt{ } s$ and $-t$ values to gain a more complete understanding of the kinematic dependence of the wave function and the relative importance of the two primary mechanisms in elastic hard scattering.

\subsection{Helicity Conservation}

The applicability of perturbative QCD (PQCD) has been controversial in exclusive reactions. ${ }^{14}$ PQCD provides a mechanism for calculating both spin-averaged and spinweighted observables. Since current quark masses are small and the QCD Lagrangian is chiral invariant, quarks do not flip helicity. Thus, on the quark level, there is no spin-flip $\left(\Phi_{5}=\Phi_{2}=0\right)$ and thus, no net polarization. However, measurements of the polarization asymmetry $A_{N}$ yield non-zero results. ${ }^{2,42}$ The implication here is that non-perturbative effects can allow for helicity flip mechanisms on the hadronic level.

We can use the kinematic dependence of the helicity amplitudes to make specific predictions about the polarization asymmetry $A_{N}$. The helicity flip amplitudes $\Phi_{2}$ and $\Phi_{5}$ for $p p \rightarrow p p$ can be constructed from the helicity conserving ones using time reversal invariance and parity arguments ${ }^{35}$ :

$$
\begin{aligned}
& \Phi_{5}(s, t) \approx[f(t)-f(u)] \Phi_{1}(s, t)+[f(t)+f(u)]\left[\Phi_{3}(s, t)+\Phi_{4}(s, t)\right], \\
& \Phi_{2}(s, t) \approx \frac{1}{2}[f(t)-f(u)] \Phi_{5}(s, t) .
\end{aligned}
$$

Here $f(t)$ and $f(u)$ are spin-flip terms which have asymptotic behavior characteristic of Regge processes at small $-t^{43}$ and higher twist processes ${ }^{44}$. Since the helicity conserving 
amplitudes have well known kinematic dependence on the Mandelstam variables, we can make specific predictions in the kinematic regions where experiments can be performed. Results are published in the reference 35 papers. The key prediction is that if helicity non-conserving effects on the hadronic level are present, we should see significant non-zero polarizations $\left(A_{N}\right)$ in two separate regions: (i) fixed angle $\left(\frac{-t}{s}\right)$ at high energies (large $\sqrt{ } s$ ) and (ii) in the hard scattering regime, $m_{p}^{2} \ll-t \ll s$. Most of the experiments outlined in section 5 can test this important aspect of QCD in these various kinematic regions.

\subsection{NPQCD Effects}

Other non-perturbative effects, such as confinement, can be investigated through exclusive processes. Some of the existing data may imply that these effects are significant, even in kinematic regions which have large enough momentum transfer $(-t)$ for QCD to normally be applicable. For example, at $90^{\circ} \mathrm{c} . \mathrm{m}$., the transverse asymmetry $A_{N N}$ exhibits oscillations about the quark model prediction of $\left(\frac{1}{3}\right){ }^{4}$ There have been many possible explanations for this behavior, ${ }^{35,45}$ including Baryon resonances (Brodsky), interferences between Landshoff and QIM amplitudes (Carlson; Ramsey) and oscillating $s$ dependence of the amplitudes (Pire). It is still not obvious whether non-perturbative effects are present, but a recent analysis of cross section data and the $A_{N N}$ data together in this kinematic region, indicates that it is likely that they are. ${ }^{35(1994)}$ Experiments could be performed at an accelerator like the Brookhaven AGS, that would extend the kinematic region of the data. Since each of the models listed above make predictions in the other kinematic regions, this data could possibly clarify the nature of this phenomenon.

Other non-perturbative effects which determine the collective properties of the proton are those which are probed at low momentum transfers. These include Instanton effects ${ }^{46}$ and the behavior of Pomerons in this region. ${ }^{43}$ These kinematic regions will likely be probed by the experiments outlined in the next section and will provide a more complete picture of the confinement of the proton and neutron constituents.

\section{Experimental Overview}

\subsection{Introduction}

We have seen that the existing data have enabled us to formulate appropriate questions which probe the spin properties of nucleons. However, there are a number of questions which remain unanswered and will only be accessible with more data at different energies and momentum transfers. Fortunately, there are a number of experimental groups that are planning polarized beam experiments at existing accelerators. With recent advances in polarized beam, target and detector technology, it is now possible to do these experiments at 
different energies and momenta in order to study the physics over a large kinematic range. ${ }^{47}$ In the following section, I will discuss some of the proposed experiments related to the spin structure of nucleons, in light of the foregoing questions presented in the last sections.

\subsection{Proposed Experiments}

The experiments discussed here represent a sampling of those which directly relate to the subject of the spin structure of nucleons. Many of these are just a small fraction of the polarization experiments which can be performed at these accelerators, but they form an integral part of the proposal. The listing below is constructed approximately in the proposed chronological order of the planned work. Table 2 lists the main polarized experiments with the energy ranges and luminosities which are reported in the proposals. The types of experiments and the important variables to be measured are included in Table 3.

\section{Table 2}

$\begin{array}{ccc}\frac{\text { Experiment }}{\text { Energy Range } \sqrt{ } \mathrm{s}(\mathrm{GeV} / \mathrm{c})} & \frac{\text { Luminosity }\left(\mathrm{cm}^{-2} \mathrm{~s}^{-1}\right)}{30(e) \text { on } 820(\mathrm{p})} & 2 \cdot 10^{31} \\ \text { HERES at HERA } & 120,1000,2000 & 2 \cdot 10^{30} \rightarrow 10^{34} \\ \text { SPIN at FNAL } & 60 \rightarrow 500 & 2 \cdot 10^{32} \\ \text { RHIC at Brookhaven } & 20 & 1 \cdot 10^{32} \\ \text { LISS at Indiana } & 120 & 1 \cdot 10^{32} \\ \text { LHC at CERN } & 400 & 1 \cdot 10^{32}\end{array}$




\section{Table 3}

\begin{tabular}{ccc} 
Experiment & Proposed Type & Measured Quantities \\
\cline { 2 - 3 } HERMES & Deep InelasticScattering & $A_{1}^{p}, g_{1}^{p}, \Delta q_{v}$ \\
SPIN & Elasticpp & $A_{N}, A_{N N}, A_{L L}$ \\
SPIN & Inelastic: jets & $\Delta G: \Delta \sigma_{L}, A_{N N}, A_{L L}$ \\
RHIC & Elastic pp, $p \bar{p}$ & $A_{N N}, A_{L L}$ \\
$R H I C$ & Inelasticjet, $\pi, \gamma$ & $\Delta G ; \Delta \sigma_{L}, A_{L L}$ \\
RHIC & DrellYan & $\Delta S$ \\
LISS & Inelastic & $\sigma_{L}, \sigma_{T}, \Delta \sigma_{L}, \Delta G$ \\
LISS & Elastic & $A_{N}, A_{L}$ \\
LHC & Elastic & $A_{N}, A_{L}, A_{N N}, A_{L L}$ \\
LHC & Inlastic & $A_{N}, A_{L}, A_{N N}, A_{L L}$ \\
$N E P T U N-A$ & Elastic $p p$ & $A_{N}, \frac{d \sigma}{d t}$
\end{tabular}

The latest proposed experiment at HERA in Hamburg plans to accelerate a large flux of polarized electrons from the storage ring and collide them with a gaseous target. ${ }^{48}$ The HERMES detector at HERA is designed to take data from the deep-inelastic scattering experiment at a large range of $x$ values, thus reinforcing the SMC and E142/3 data. The gaseous target will eliminate some of the systematic errors characteristic of solid targets, which were used in the other experiments. With lower error bars at the small $x$ values, it should be easier to extrapolate $g_{1}^{p}(x)$ and achieve a more accurate integrated value, $\left\langle g_{1}^{p}\right\rangle$. Thus, comparison to the Ellis-Jaffe sum rule will be more accurate as will the ability to determine the polarized sea values from this data.

The SPIN Collaboration has proposed to do fixed target $p p$ and $p \bar{p}$ experiments at energies of $120 \mathrm{GeV}$ and $1 \mathrm{TeV}$ at Fermilab. ${ }^{49}$ The proposal also includes $p p$ collider experiments at $2 \mathrm{TeV}$. The higher luminosity listed would be at the lowest energies. One of the crucial contributions that this set of experiments can make is the extremely large range of high $p_{T}$ that can be covered. This set of experiments is in the kinematic region where the measurement of double spin asymmetries in jet production would give a sensitive test of the polarized gluon distribution's size. Naturally, this has an effect on both $\Delta G$ and the anomaly term appearing in the polarized quark distributions. The high $p_{T}$ measurements of the elastic single and double spin asymmetries would be helpful in determining the parameters of helicity non-conservation $(f(t))$ in the fixed $-t$, large $s$ region. Fixed small angle scattering at large $-t$ and $s$ would determine the relative contributions of the QIM and Landshoff diagrams 
mentioned in section 4 .

The Relativistic Heavy Ion Collider (RHIC) at Brookhaven is designed to be an accelerator of both light and heavy ions. The high energy community has proposed that polarized $p p$ and $p \bar{p}$ experiments be performed, due to the large energy and momentum transfer ranges which should be available. ${ }^{50}$ The energy range in Table 2 will be made in discrete steps of about 60,250 and $500 \mathrm{GeV}$, but the momentum transfer range covers $0.005 \leq-t \leq 6.0$ $\mathrm{GeV}^{2}$ in a fairly complete set of steps. There are two main proposed detectors, STAR and PHENIX, which have different but complementing capabilities. Between the two detector groups, most of the experiments mentioned in sections 3 and 4 can be performed at RHIC. The energies and momentum transfers would be complementary to those at Fermilab and would provide a wealth of data. Inclusive reactions involving jet measurements, direct photon production and pion production would be excellent tests of the $Q^{2}$ dependence of $\Delta G$ since all of these are sensitive to the polarized gluon density at differing $Q^{2}$ values. Polarized Drell-Yan experiments are also planned, which would give reasonable estimates to the polarized sea for each flavor. Elastic scattering, especially at the larger $-t$ range could shed light on both helicity non-conservation at the hadronic level and non-perturbative effects discussed in section 4. Measurement of the transverse spin and transversity distributions would probe higher-twist non-perturbative effects as well.

Recently, a proposal for a new light ion accelerator has been announced, which will specialize in polarization experiments. ${ }^{51}$ The Light Ion Spin Synchrotron (LISS) would be located in Indiana, and would perform a variety of polarization experiments for both high energy and nuclear physics. The energy range would be lower that most other experiments, thus complementing the kinematic areas covered. Furthermore, both proton and deuteron beams could be available to perform inclusive scattering experiments. They propose to measure longitudinal and transverse cross sections and spin asymmetries which will address the wave function normalization problem previously discussed. Elastic scattering measurements of $A_{N}$ at small -t could give valuable information regarding helicity non-conservation in this region.

The CERN proposal for polarization physics at the LHC indicates that the characteristics which make that facility unique for spin physics include: (i) a jet target with $90 \%$ polarization; (ii) a large energy range not covered by other experiments and (iii) the ability to reach large momentum transfers (to $-t=10 \mathrm{GeV}^{2}$ ). ${ }^{52}$ The plan is to perform a variety of inclusive and exclusive polarization measurements, including transverse spin asymmetries. These would likely complement the other experiments mentioned above.

The NEPTUN-A collaboration at Serpukhov plans to perform elastic $p p$ experiments at very large $s$. They would measure elastic differential cross sections (with high luminosity beams for good event statistics) and $A_{N}$ at large $p_{T}^{2}$. This is an excellent large $s$ probe of 
the fixed $-t$ regions to investigate helicity non-conservation and applicability of QCD to exclusive processes.

\section{Conclusion}

The property of spin provides us with a unique way of probing hadronic structure. Existing data has indicated that the spin structure of nucleons is non-trivial and has led to the formulation of a crucial set of questions to be answered about this structure. Theoretical work in this area has provided links of these spin questions to some of the most fundamental tests of QCD, such as chiral invariance, helicity non-conservation and even the quark and gluon structure of hadrons. There are many proposals for future polarization experiments, which complementarily will provide answers to some of these questions. When the data of these experiments is analyzed, we will be able to extract a wealth of fundamental physical information from them.

\section{References}

1. J. Ashman, et.al., Phys. Lett. B206, 364 (1988); Nucl. Phys. B328, 1 (1989).

2. G.W. Abshire, et. al., Phys. Rev. Lett. 32, 1261 (1974).

3. M.J. Alguard, et. al., Phys. Rev. Lett. 37, 1261 (1976); G. Baum, et.al., Phys. Rev. Lett. 51, 1135 (1983).

4. A. Lin, et. al., Phys. Lett. 74B, 273 (1978) and E.A. Crosbie, et. al., Phys. Rev. D23, 600 (1981).

5. A.D. Krisch, Sci. Am. 257(2), 42 (1987, Aug.)

6. J.D. Bjorken Phys. Rev. 148, 1467 (1966).

7. J. Ellis and R.L. Jaffe, Phys. Rev. D9 (1974) 3594.

8. B. Adeva, et. al., Phys. Lett. B302, 533 (1993) and Phys. Lett. B320, 400 (1994); D. Adams, et. al., Phys. Lett. B329, 399 (1994).

9. P.L. Anthony, et. al., Phys. Rev. Lett. 71, 959 (1993); K. Abe, et. al., SLAC preprint 6508 (1994), submitted to Phys. Rev. Lett. .

10. D.L. Adams, et al., (FNAL E581/704 Collaboration), Phys. Lett. B336, 269 (1994). 
11. R.C. Kammerud, et. al., Phys. Rev. D4, 1309 (1971); C.W. Akerlof, et. al., Phys. Rev. 159, 1138 (1967).

12. Foley, et.al., Phys. Rev. Lett. 15, 45 (1965).

13. A. Bohm, et. al., Phys. Lett. 49B, 491 (1974); E. Nagy, et. al., Nucl. Phys. B150, 221 (1979); J.V. Allaby, et.al., Phys. Lett. 28B, 69 (1968).

14. N. Isgur and C.H. Llewellyn-Smith, Phys. Rev. Lett. 52, 1080 (1984).

15. G. Ramsey, et.al., Phys. Rev. D39, 361 (1989); J.-W. Qiu, et.al., Phys. Rev. D41, 65 (1990).

16. R.L. Jaffe Phys. Lett. B193, 101 (1987).

17. Carlitz and Kaur Phys. Rev. Lett. 38, 673 (1977).

18. Chiapetta and Soffer, Phys. Rev. D31, 1019 (1985); Einhorn and Soffer, Nucl. Phys. B274, 714 (1986)

19. H.J. Lipkin, Phys. Lett. B251, 613 (1990); B.-A. Li, et. al., Phys. Rev. D43, 1515 (1991).

20. Close and Sivers, Phys. Rev. Lett. 39, 1116 (1977).

21. S.J. Brodsky and G.R. Farrar, Phys. Rev. Lett. 31, 1153 (1973) and Phys. Rev. D11, 1309 (1975); V.A. Matveev, R.M. Muradyan and A.V. Tavkheldize, Lett. Nuovo Cimento 7, 719 (1973); A.W. Hendry, Phys. Rev. D10, 2300 (1974).

22. P. Amaudruz, et.al, Phys. Rev. Lett. 66, 2712 (1991).

23. See for example, F.E. Close and R.G. Roberts, Phys. Lett. B316, 165 (1993).

24. H.J. Lipkin, Phys. Lett. B214, 429 (1988) and Weizmann Preprint WIS-94/24/May$\mathrm{PH}$ (1994).

25. F.E. Close and R.G. Roberts, Phys. Rev. Lett. 60, 1471 (1988); S.J. Brodsky, J. Ellis and M. Karliner, Phys. Lett. B206, 309 (1988); M. Anselmino, B.L. Ioffe, and E. Leader Yad. Fiz. 49, 214 (1989); G. Preparata, P.G. Ratcliffe and J. Soffer, Phys. Lett. B273, 306 (1991); H.J.Lipkin, Phys. Lett. B256, 284 (1991).

26. M. Goshtasbpour and G.P. Ramsey, ANL-HEP-PR-94-90.

27. H.-Y. Cheng and S.-N. Lai, Phys. Rev. D41, 91 (1990). 
28. G.P. Ramsey and D. Sivers, Phys. Rev. D43, 2861 (1991).

29. G.P. Ramsey, D. Richards and D. Sivers Phys. Rev. D37, 314 (1988).

30. Bourrely, J. Soffer and P. Taxial, Phys. Rev. D36, 3373 (1987).

31. E.L. Berger and J.-W. Qiu, Phys. Rev. D40, 778 (1989); P.M. Nadolsky, Z. Phys. C62, 109 (1994).

32. J. Babcock, E. Monsay and D. Sivers, Phys. Rev. Lett. 40, 1161 (1978) and Phys. Rev. D19, 1483 (1972).

33. A.V. Efremov and O.V. Teryaev, JINR Report E2-88-287 (1988); G. Alterelli and G.G. Ross, Phys. Lett. B212, 391 (1988); R.D. Carlitz, J.C. Collins, and A.H. Mueller, Phys. Lett. B214, 229 (1988).

34. S.E. Troshin and N.E. Tyurin, Particle World 3, 165 (1993).

35. G. Ramsey and D. Sivers Phys. Rev. D45, 79 (1992), Phys. Rev. D47, 93 (1993) and ANL-HEP-PR-94-69 (1994).

36. G.P. Lepage and S.J. Brodsky, Phys. Rev. D22, 2157 (1980) and S. Brodsky, et.al., Phys. Lett. 91B, 239 (1980).

37. P.V. Landshoff, Phys. Rev. D10, 1024 (1974); Landshoff and D.J. Pritchard, Z.Phys. C6, 69 (1980).

38. A. Donnachie and P.V. Landshoff., Z. Phys. C 2, 55 (1979); Phys. Lett. 123B, 345 (1983); Nucl. Phys. B231, 189 (1983); B244, 322 (1984); B267, 690 (1986).

39. J. Botts, Nucl. Phys. B353, 20 (1991) and Phys. Rev. D44, 2768 (1991).

40. M. Jacob and G.C. Wick, Ann. Phys. 7, 404 (1959).

41. G. Farrar, S. Gottlieb, D. Sivers and G. Thomas, Phys. Rev. D20, 202 (1979) and S.J. Brodsky, C.E. Carlson and H.J. Lipkin, Phys. Rev. D20, 2278 (1979).

42. P.R. Cameron et. al., Phys. Rev. D32, 3070 (1985).

43. M. Block, K. Kang and A. White, Int. J. Mod. Phys., A7, 4449 (i992).

44. See for example, R.L. Jaffe, in Proceedings of the 10th International Symposium on High Energy Spin Physics, (Nagoya, Nov, 1992), c1993, Universal Academy Press, Inc., Tokyo. 
45. S.J. Brodsky and G. deTeramond, Phys. Rev. Lett. 60, 1924 (1988); B. Pire and J.P. Ralston, Phys. Lett. 117B, 233 (1982); C.E. Carlson, M. Chachkhunashvili and F. Myher, Phys. Rev. D46, 2891 (1992).

46. A.E. Dorokhov, et. al., Phys. Lett. B304, 167 (1993).

47. S.B. Nurushev, IHEP preprint IHEP 91-103, Protvino, Russia. See also papers in the Proceedings of the 10th International Symposium on High Energy Spin Physics, (Nagoya, Nov, 1992), c1993, Universal Academy Press, Inc., Tokyo, on the following topics: Polarized Beams-Snakes (Phelps, Roser, SY Lee), Luminosity (Tanaka, Mori); Polarized Targets-Solid (Durand, vanden Brandt), Gaseous (Haeberli, Kaufman).

48. Physics News brief: "Polarization at HERMES", Phys. Today, 47(11), 19 (1994(Nov.)).

49. SPIN Collaboration, A.D. Krisch, et. al., "Expression of Interest: Accelerated Polarized Beam Experiments at the Fermilab Tevatron", May, 1994.

50. RHIC Spin Collaboration, "Proposal on Spin Physics Using the RHIC Polarized Collider", August, 1992; and STAR-RSC Update, October, 1993.

51. J.M. Cameron and S. Vigdor, "The LISS Brief", private communication.

52. LHC Polarization Proposal, 1994.

53. NEPTUN-A collaboration, "Spin Proposal for Experiments at Serpukhov", 1993. 